\title{
Low creatine kinase is associated with a high population incidence of fainting
}

\author{
Lizzy M. Brewster · Gideon Mairuhu · Karin Ganzeboom • Nynke van Dijk • \\ Gert A. van Montfrans · Wouter Wieling
}

Received: 30 September 2008/ Accepted: 3 April 2009/Published online: 26 May 2009

(C) The Author(s) 2009. This article is published with open access at Springerlink.com

\begin{abstract}
Objective Vasoconstrictor capacity, skeletal muscle tone, and renal sodium retention are involved in the pathogenesis of fainting. As muscle contractility and ion transport are highly energy-demanding processes, we hypothesized that a low activity of the energy-generating enzyme creatine kinase (CK) is associated with a higher risk of fainting. The aim of this observational study was to explore the association of vasovagal syncope with low CK.

Methods A random sample of 1,000 subjects aged 34-60 years was drawn from the general population, with 442 subjects eventually included in the study. Data on fainting history were collected with the investigators blinded to participants' CK level. We prepared this report according to the "Strengthening the Reporting of Observational Studies in Epidemiology" (STROBE) statement. The main outcome was the lifetime cumulative incidence
\end{abstract}

L. M. Brewster · K. Ganzeboom · G. A. van Montfrans .

W. Wieling

Department of Internal Medicine, Academic Medical Center, University of Amsterdam, F4-222, Meibergdreef 9,

1105 AZ Amsterdam, The Netherlands

L. M. Brewster $(\varangle) \cdot$ G. A. van Montfrans

Department of Vascular Medicine, Academic Medical Center,

University of Amsterdam, F4-222, Meibergdreef 9,

1105 AZ Amsterdam, The Netherlands

e-mail: 1.m.brewster@amc.uva.nl

N. van Dijk

Department of Clinical Epidemiology and Biostatistics,

Academic Medical Center, University of Amsterdam,

Meibergdreef 9, 1105 AZ Amsterdam, The Netherlands

G. Mairuhu

Department of Cardiology, St Antonius Hospital, Koekoekslaan

1, POB 2500, 3430 EM Nieuwegein, The Netherlands of vasovagal syncope in subjects with low versus highnormal serum CK after a 3 days rest.

Results The proportion of fainters within the high CK group was 29 out of $130(22 \%)$ versus 121 out of 312 (39\%) in the low CK group; a $73 \%$ greater occurrence of fainting with low CK $(P=0.0005)$. This finding was consistent across recurrent fainters, and in men and women.

Interpretation Low CK is associated with a $73 \%$ higher incidence of fainting in a random population sample. The association is biologically plausible, as CK enhances cardiovascular and skeletal muscle contractility and salt retention. The presented data suggest that low CK activity is a potential new risk factor for vasovagal syncope.

Keywords Vasovagal syncope $\cdot$ Fainting ·

Creatine kinase $\cdot$ ATP $\cdot$ Energy metabolism

\section{Introduction}

We proposed that creatine kinase (CK), the central regulatory enzyme of energy metabolism, may increase orthostatic tolerance. The enzyme catalyzes the reversible transfer of the high-energy phosphate moiety $(\mathrm{P})$ between creatine and ADP:

$\operatorname{MgADP}+$ PCreatine $+\mathrm{H}^{+} \leftrightarrow \operatorname{MgATP}+$ Creatine,

with a capacity to regenerate ATP at a higher rate than glycolysis and oxidative phosphorylation together $[4,5,8$, $10,27,29]$. CK is bound near ion transporters in cellular membranes including $\mathrm{Na}^{+} / \mathrm{K}^{+}$-ATPase and $\mathrm{Ca}^{2+}$-ATPase. In addition, the enzyme is active near myosin light chain kinase and myosin ATPase, the principal enzymes involved in muscle contraction $[4,5,8,10,27,29]$. CK thus fuels the 
maintenance and restoration of the membrane potential, of renal sodium retention, and of cardiovascular and skeletal muscle contractility [2, 4, 5, 8, 10, 14, 17, 27, 29].

Vasovagal syncope refers to a sudden loss of consciousness with a loss of postural tone, due to a rapid fall in blood pressure and cerebral perfusion, resulting from reflex vasodilation and bradycardia. The condition, which can be debilitating when it occurs recurrently, is provoked by diverse stimuli including strong emotion, Valsalva maneuvers, and prolonged standing [9, 11, 12, 15, 18, 24]. A diminished vasoconstrictor capacity, reduced circulating blood volume, and decreased skeletal muscle tone have been identified as causal factors, but it remains unclear why some people faint more frequently than others $[9,11,12$, 15, 18, 24].

We hypothesized that subjects with a low CK activity have a lower cellular energetic capacity to withstand orthostatic and other stresses that lead to fainting. In this observational study, we assessed in a random population sample, whether the cumulative incidence of vasovagal syncope is higher with low CK.

\section{Methods}

In preparing this report, we followed the STROBE (The Strengthening the Reporting of Observational Studies in Epidemiology) Statement, a guideline intended to protocol and improve the reporting of observational studies [28].

\section{Study population}

We analyzed a random sample of the white population participating in the Cardiovascular Risk profile of native Dutch people in the Netherlands (CRANS) study, a sister study to the "SUrinamese in the Netherlands: Study on EThnicity and health" (SUNSET) Study [5], crosssectional surveys on cardiovascular risk factors of people living in Amsterdam, the Netherlands, conducted from 2001 to 2003. The institutional review committee approved the study and the participants gave written informed consent.

The methods have been previously reported $[5,6]$. In brief, registration offices of the selected municipalities in Amsterdam provided the CRANS study group a stratified random population sample of 1,000 non-institutionalized white persons aged 34-60 years. The overall response rate was $57 \%$. All prospective participants were invited for an oral interview. The respondents were interviewed at home by trained study-personnel. The questionnaire included demographic data, cardiovascular health status, and risk factors including life style, diet and smoking, and history of syncope.
Incidence of syncope

During a visit at home, data were collected on the selfreported occurrence of vasovagal syncope, as defined by the European Society of Cardiology [9]. This structured interview included the occurrence of loss of consciousness, and if applicable, the frequency of these episodes, the age at the first episode, subsequent occurrences if any, and the perceived trigger of the episodes, as previously described [9, 13, 20, 21]. Participants were specifically questioned regarding the presence of diagnosed seizure disorders, diagnosed cardiac disease, and other possible causes of a loss of consciousness, as recommended [9, 13, 20, 21].

\section{Physical examination}

All participants were invited to the hospital for a physical examination. We instructed participants to abstain from heavy exercise during 3 days before visiting our hospital. Walking, driving, and normal daily activities were allowed. Physical examination included height, weight, and blood pressure levels. Blood pressure was measured in the morning in all participants, in a quiet room in the sitting position, with an Omron M4 oscillometric device (Omron Healthcare Europe B.V., Hoofddorp, The Netherlands). An appropriately adjusted cuff size was used on the nondominant arm, which was supported at heart level. To account for blood pressure variability, we used the mean of the first two consecutive readings with a maximum of $5 \mathrm{mmHg}$ difference [5].

\section{Serum CK estimations}

Laboratory studies included serum CK activity after 3 days of rest, as a measure of tissue $\mathrm{CK}$ activity as previously described [5]. In otherwise healthy people, the release of the enzyme from tissue at rest is proportional to the level of tissue CK activity [5]. CK activity was estimated with automated analyzers (Roche/Hitachi Systems, Roche Diagnostics, Indianapolis, IN, USA), according to the procedure recommended by the International Federation of Clinical Chemistry [25].

\section{Exclusion criteria}

Exclusion criteria for our analyses included seizure disorders and syncope with an established cause, such as intoxication or cardiac disease [9, 13]. We also excluded participants with treated hypertension, as postural hypotension might be a side effect of antihypertensive medication [9]. 
Statistical analysis

\section{Outcome}

The primary outcome was the difference in lifetime cumulative incidence of vasovagal syncope between subjects with low versus high CK activity. We opted to dichotomize the data (low vs. high CK) around the population median for serum CK activity for non-black people $(<111 \mathrm{IU} / \mathrm{l} \mathrm{vs} . \geq 111 \mathrm{IU} / \mathrm{l})$, as previously reported by our group [6]. We performed a sensitivity analysis and recalculated the primary outcome post hoc using tertiles for CK based on population data, with similar results (data not shown).

As a secondary outcome, we analyzed the primary outcome in participants with and without recurrent fainting; and in men and women. The tertiary outcome was the first-order partial correlation between a history of fainting, and significant correlates of this outcome, controlling for $\log \mathrm{CK}$ activity as a continuous measure.

\section{Sample size calculation}

Based on differences in syncope incidence between ethnic subgroups known to differ in serum CK activity [6, 20, 21], and the expected greater proportion of white subjects in the predefined low CK group [5, 6], we estimated to find a 50\% higher syncope rate with low-normal CK, and calculated we needed 209 subjects in the low CK group and 105 in the high CK group to detect this difference with two-tailed $\alpha=0.05$ and $1-\beta=0.80$.

\section{Analyses}

Lifetime cumulative incidence was calculated as the proportion of individuals who had at least one episode of vasovagal syncope during their life $[9,13]$. Because of the skewed distribution of CK, we performed a log transformation to the base of ten on the CK data, and excluded data above the 97.5 percentile [5, 6]. Differences in study parameters between the low and high CK group were calculated with Fisher's exact test for fainting and sex, and with the unpaired $t$ test for the continuous measures age, systolic blood pressure (SBP), body mass index (BMI), and $\mathrm{CK}$, before calculating the proportional difference in the cumulative rate of fainting with low versus high-normal $\mathrm{CK}$ activity as the primary outcome. Confidence intervals for the proportions were estimated with the adjusted Wald method.

As $\mathrm{CK}$ is proposed to be an intervening variable that mediates the effect of sex and obesity on cardiovascular function [7], and a causal factor affecting blood pressure [5], we assessed to which extent the continuous measure $\log$ CK explained the effect of these parameters on fainting history with partial correlation analysis, which is commonly used in "causal" modeling of small models with three to five variables [7]. Because we collected data on the history of fainting in subjects aged 34-60 years, we did not include the variable "current age" into the correlation analyses.

Data in square brackets are $95 \%$ confidence intervals, unless otherwise specified. Where applicable, we considered a one-sided $P$ value of $<0.05$ to be significant, under the assumption that subjects with high CK activity would have a lower occurrence of fainting. Main statistical analyses were performed with SPSS statistical software package for Windows, version 14.0 (SPSS Inc., Chicago, IL, USA) and the Web-based computation facilities at http:// faculty.vassar.edu/lowry/VassarStats.html.

\section{Results}

We included 442 subjects (220 men) in the study. Participants' flow is depicted in Fig. 1. Table 1 shows the summary data of the subjects with high versus low CK (as defined in Sect. "Methods") [6].

A total of 150 participants (34\%) ever fainted during their lifetime. The proportion of fainters within the high

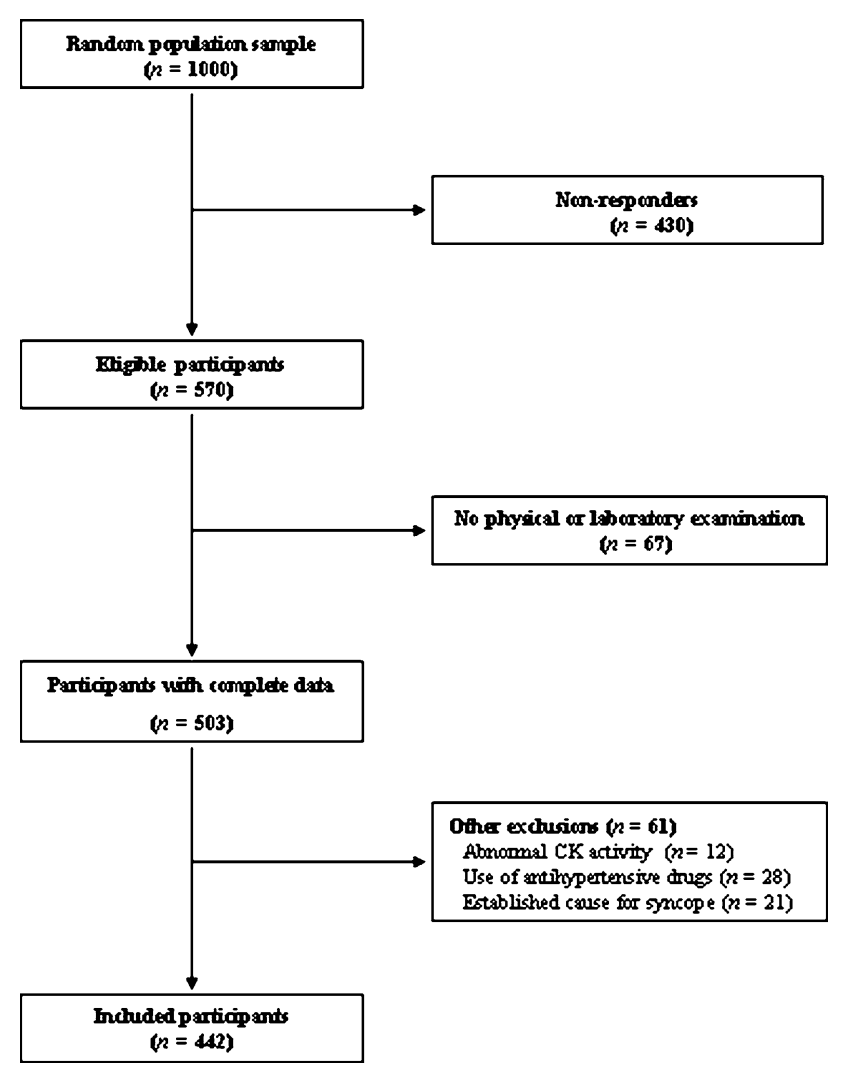

Fig. 1 Participants' flow 
Table 1 Study parameters with high and low CK

$S B P$ systolic blood pressure, $B M I$ body mass index; $C K$ creatine kinase activity

$* P<0.05$ for differences in mean values between low and high $\mathrm{CK}$ groups

\begin{tabular}{llll}
\hline Study parameters & High CK & Low CK & Total \\
\hline$N$ (men) & $130(104)$ & $312(116)$ & $442(220)$ \\
Age, years (SE) & $47.9(0.6)$ & $47.4(0.4)$ & $47.6(0.3)$ \\
SBP, mmHg (SE)* & $128.1(1.7)$ & $120.9(1.1)$ & $123.0(0.9)$ \\
BMI, kg/m²(SE) & $26.4(0.4)$ & $25.7(0.3)$ & $25.9(0.2)$ \\
$\mathrm{CK}, \mathrm{IU} / \mathrm{L}(\mathrm{SE})^{*}$ & $161.5(4.1)$ & $72.1(1.2)$ & $98.4(2.4)$ \\
History of fainting $(N)^{*}$ & 29 & 121 & 150 \\
Recurrent fainting $(N)^{*}$ & 15 & 81 & 96 \\
Age at first fainting episode, years $(\mathrm{SE})^{*}$ & $24(2.3)$ & $19(1.0)$ & $21(0.9)$
\end{tabular}

CK group was 29 out of 130 (22\%), versus 121 out of 312 (39\%) within the low CK group, an increase in fainters of $73 \%$ with low CK activity [OR 2.20 (95\% CI 1.38-3.54), $P=0.0005]$.

Furthermore, 96 subjects were recurrent fainters (2 fainting episodes or more, median 4 episodes), respectively, 15 out of 130 (12\%) in the high CK group, versus 81 out of $312(26 \%)$ with low CK, an increase in recurrent fainters of $117 \%$ with low CK [OR 2.67 (95\% CI 1.484.88), $P=0.0004]$.

In line with this, the proportion of high CK subjects decreased in subgroups with increasing fainting frequency, respectively, 35, 26, and $15 \%$, for never, one-time, and multiple fainters (one-way ANOVA, $P<0.001$; Fig. 2).

It is known that women tend to have a lower mean CK activity than men $[4,6]$. Women are also known to have a greater tendency to faint $[13,20,21]$. Therefore, we reassessed the results separately for subgroups of women and men. The cut-off point in women for high versus low occurrence of fainting was observed around a CK of

Fainting episode frequency and resting serum CK
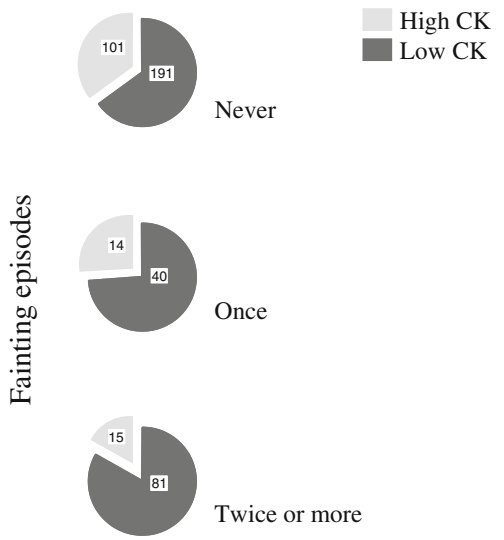

Fig. 2 Fainting episode frequency and resting serum CK. The figure shows a decreasing proportion of high $\mathrm{CK}$ subjects in categories of increasing fainting episodes, respectively, 35, 26, and 15\%, for never, one-time, and multiple fainters (one-way ANOVA, $P<0.001$ ). The numbers depicted in the circular chart denote the number of subjects in each group
107 IU/l, with a drop in the percentage of fainters from $44 \%(88 / 199)$ below this cut-off point, to $11 \%(4 / 35)$ fainters with a CK activity $\geq 107$ IU/l, an OR of 6.14 (95\% CI 2.09-18.06) for fainting with low CK. For men, the cutoff point was higher, at $130 \mathrm{IU} / \mathrm{l}$, with a percentage of fainters of $45 \%$ (74/164) below this cut-off point, versus $18 \%(13 / 72)$ fainters with a CK activity $\geq 130 \mathrm{IU} / 1$, an OR of 4.49 (95\% CI 2.36-8.53) $(P<0.001$ for differences in both groups).

Finally, as $\mathrm{CK}$ is proposed to be an intervening variable between sex and BMI versus the outcome fainting, and a causal factor affecting blood pressure [5, 7], we controlled for $\mathrm{CK}$ as a continuous measure in first-order partial correlation analysis [7]. When controlling for $\mathrm{CK}$, the small but significant negative correlations between a history of fainting and male sex, SBP, and BMI (squared correlation coefficients, respectively, 1.23, 1.77, and 1.17; $P<0.001$ ), showed a $55 \%$ reduction for sex, a $20 \%$ reduction for blood pressure, and a $13 \%$ reduction for BMI, whereas the correlation of fainting with sex was rendered insignificant.

\section{Discussion}

In this observational study, we explored in a random population sample the association between CK and fainting, and provide data indicating that men and women with low CK faint more often than with high CK. In addition, those with low CK experience their first fainting episode at a younger age. Moreover, the correlation analysis used because CK is proposed to be a causal factor [7], indicated that controlling for $\mathrm{CK}$ reduces the effect of blood pressure and BMI on fainting, and renders the correlation of fainting with sex insignificant.

Our observational data are in line with the observation that subgroups of the population with high tissue and serum CK, including men, obese people and black people, display greater orthostatic tolerance [2-6, 9, 13, 16, 20-23, 25, 26]. However, our data cannot be considered proof of a causal role for $\mathrm{CK}$, although the evidence from previous studies shows that $\mathrm{CK}$ has a central role in muscular contractility. 
The increase in peripheral vascular resistance is the principal method by which blood pressure is maintained during orthostatic stress $[12,15,24]$. Therefore, the ability to tolerate orthostatic stress is directly related to the ability to increase vascular resistance, coined "vasoconstrictive reserve" $[12,15,24]$. We showed that vascular contractility is CK-dependent in humans [8]. Therefore, high tissue CK may augment cellular energetic capacity to withstand gravitational and other stresses that provoke syncope [9, 11, 12, 15, 18, 24], through enhancing vascular tone and vasoconstrictor reserve [4, 5, 8]. Subjects with high $\mathrm{CK}$ might thus have greater energy reserve to rapidly respond to threatening hypotension, mainly through a greater ability to suddenly increase peripheral vascular resistance, resulting in a better protection of cerebral perfusion pressure. Furthermore, relatively high skeletal muscle CK activity is associated with a predominance of fast type 2 fibers and anaerobic muscle metabolism capacity, with capillary rarefaction reducing the peripheral capacitance of vessels [19]. High muscle CK thus helps to generate swift skeletal muscle responses, with less pooling of blood, and greater vascular peripheral resistance. Finally, $\mathrm{CK}$ is thought to fuel sodium retention in the kidney, with a high $\mathrm{CK}$ leading to greater salt retaining capacity [14, 17]. Therefore, low CK might decrease orthostatic tolerance.

This observational study has some clinical and methodological limitations. The diagnosis of vasovagal syncope in this population study was based on history taking and general physical examination. Although we excluded patients with diagnosed cardiac or neurological diseases, we might have missed syncope secondary to undiagnosed cardiac and other disorders. However, subjects typically had an incidence peak of syncope around the age of 20 years [13]. Compared to the $35 \%$ incidence of syncope in our participants, the prevalence of epileptic seizures in a similar young age group is much lower (less than 1\%) and syncope resulting from cardiac arrhythmias or structural heart disease, i.e., cardiac syncope, is even less common [9], and more importantly, not likely to systematically differ between low and high CK groups, or to impact our outcome. Furthermore, our study has a semi-cross-sectional design in which current $\mathrm{CK}$ is compared to a history of prior fainting, which may be subject to recall bias, although this also should not systematically differ between high and low CK groups. However, the design does create limitations regarding the interpretation of the data. This study should be considered to be hypothesis-generating [28], providing initial data that suggest that low $\mathrm{CK}$ activity is a risk factor for fainting.

Vasovagal syncope is well known in medical history and in the public perception [1]. Because of its common occurrence $[9,11-13,15,18,24]$, it was suggested that fainting is not a disease [1]. We proposed a new causal factor that is both common and normal, the condition of relatively low CK activity [4-6, 8]. Our observational study clearly indicates that in a random population sample, low CK activity is associated with a $73 \%$ greater incidence of fainting. The finding was apparent in both men and women. In addition, we found evidence of a greater occurrence of recurrent fainting with low $\mathrm{CK}$, and the first fainting episode occurred at a younger age. The observational design of the study does not permit causal inferences, but as $\mathrm{CK}$ controls muscle contractility and renal salt retention, our data may help understand the greater occurrence of fainting in subgroups with low $\mathrm{CK}$, such as in women [9, 13]. Further studies are needed to explore the potential role of tissue energy metabolism and a low tissue energy reserve as a new risk factor for vasovagal syncope.

Acknowledgments The authors did not receive external funding for this study.

Conflict of interest statement All authors declare that they have no competing interests.

Open Access This article is distributed under the terms of the Creative Commons Attribution Noncommercial License which permits any noncommercial use, distribution, and reproduction in any medium, provided the original author(s) and source are credited.

\section{References}

1. Alboni P, Brignole M, Degli Uberti EC (2007) Is vasovagal syncope a disease? Europace 9:83-87

2. Ama PFM, Simoneau JA, Boulay MR, Serresse O, Thériault G, Bouchard C (1986) Skeletal muscle characteristics in sedentary black and Caucasian males. J Appl Physiol 61:1758-1761

3. Borges O, Essen-Gustavsson B (1989) Enzyme activities in type I and II muscle fibres of human skeletal muscle in relation to age and torque development. Acta Physiol Scand 136:29-36

4. Brewster LM, Clark JF, van Montfrans GA (2000) Is greater tissue activity of creatine kinase the genetic factor increasing hypertension risk in black people of sub-Saharan African descent? J Hypertens 18:1537-1544

5. Brewster LM, Mairuhu G, Bindraban NR, Koopmans RP, Clark JF, van Montfrans GA (2006) Creatine kinase activity is associated with blood pressure. Circulation 114:2034-2039

6. Brewster LM, van Montfrans G (2007) Distribution of creatine kinase in the general population: implications for statin therapy. Am Heart J 154:655-661

7. Brewster LM, Stronks K, Zwinderman AH, van Montfrans GA (2008) Creatine kinase and the correlates of blood pressure in a random population sample. Hypertension 51:e4-5

8. Brewster LM, Taherzadeh Z, Clark JF, van Bavel E, Wolf H, van Montfrans GA (2006) Contraction in human resistance arteries depends on creatine kinase. J Hypertens 24:S403 (Abstract)

9. Brignole M, Alboni P, Benditt D, Bergfeldt L, Blanc JJ, Bloch Thomsen PE, van Dijk JG, Fitzpatrick A, Hohnloser S, Janousek J, Kapoor W, Kenny RA, Kulakowski P, Moya A, Raviele A, 
Sutton R, Theodorakis G, Wieling W (2001) Task force on syncope, European society of cardiology. Guidelines on management (diagnosis and treatment) of syncope. Eur Heart $\mathrm{J}$ 22:1256-1306

10. Clark JF (1994) The creatine kinase system in smooth muscle. Mol Cell Biochem 133-134:221-232

11. Cooper VL, Hainsworth R (2002) Effects of dietary salt on orthostatic tolerance, blood pressure and baroreceptor sensitivity in patients with syncope. Clin Auton Res 12:236-241

12. Fu Q, Witkowski S, Levine BD (2004) Vasoconstrictor reserve and sympathetic neural control of orthostasis. Circulation 110:29312937

13. Ganzeboom KS, Mairuhu G, Reitsma JB, Linzer M, Wieling W, van Dijk N (2006) Lifetime cumulative incidence of syncope in the general population: a study of 549 Dutch subjects aged 3560 years. J Cardiovasc Electrophysiol 17:1172-1176

14. Greger R (2000) Physiology of renal sodium transport. Am J Med Sci 319:51-62

15. Hainsworth R (2004) Pathophysiology of syncope. Clin Auton Res 14:18-24

16. Hittel DS, Hathout Y, Hoffman EP, Houmard JA (2005) Proteome analysis of skeletal muscle from obese and morbidly obese women. Diabetes 54:1283-1288

17. Ikeda K (1988) Localization of brain type creatine kinase in kidney epithelial cell subpopulations in rat. Experientia 44:734735

18. Mayerson HS, Burch GE (1939) Relationships of tissue (subcutaneous and intramuscular) and venous pressures to syncope induced in man by gravity. Am J Physiol 128:258-269

19. Momken I, Fortin D, Serrurier B, Bigard X, Ventura-Clapier R, Veksler V (2002) Endothelial nitric oxide synthase (NOS) deficiency affects energy metabolism pattern in murine oxidative skeletal muscle. Biochem J 368:341-347

20. Newman BH (2007) Vasovagal reactions in high school students: findings relative to race, risk factor synergism, female sex, and non-high school participants. Transfusion 42:1557-1560

21. Newman BH, Pichette S, Pichette D, Dzaka E (2003) Adverse effects in blood donors after whole-blood donation: a study of
1000 blood donors interviewed 3 weeks after whole-blood donation. Transfusion 43:598-603

22. Ramirez O, Licea G, Santos G (1981) Special capabilities of the sexes: differences in the molecular repertoire of mammal brain, heart and skeletal muscle. Arch Invest Med 12:377-393

23. Salvadori A, Fanari P, Ruga S, Brunani A, Longhini E (1992) Creatine kinase and creatine kinase-MB isoenzyme during and after exercise testing in normal and obese young people. Chest 102:1687-1689

24. Schondorf R, Wieling W (2000) Vasoconstrictor reserve in neurally mediated syncope. Clin Auton Res 10:53-55

25. Schumann G, Bonora R, Ceriotti F, Clerc-Renaud P, Ferrero CA, Ferard G, Franck PF, Gella FJ, Hoelzel W, Jorgensen PJ, Kanno T, Kessne A, Klauker R, Kristiansen N, Lessinger JM, Linsinger TP, Misaki H, Panteghini M, Pauwels J, Schimmel HG, Vialle A, Weidemann G, Siekmann L (2007) IFCC primary reference procedures for the measurement of catalytic activity concentrations of enzymes at 37 degrees C. Part 2. Reference procedure for the measurement of catalytic concentration of creatine kinase. Clin Chem Lab Med 40:635-642

26. Shvartz E, Wyndham CH, Strydom NB (1975) Orthostatic responses in Caucasians and Bantu. Aviat Space Environ Med 46:1343-1348

27. Ventura-Clapier R, Veksler V, Hoerter JA (1994) Myofibrillar creatine kinase and cardiac contraction. Mol Cell Biochem 133134:125-144

28. Von Elm E, Altman DG, Egger M, Pocock SJ, Gøtzsche PC, Vandenbroucke JP, STROBE Initiative (2007) The Strengthening the Reporting of Observational Studies in Epidemiology (STROBE) statement: guidelines for reporting observational studies. Ann Intern Med 147:573-577

29. Wallimann T, Dolder M, Schlattner U, Eder M, Hornemann T, O'Gorman E, Ruck A, Brdiczka D (1998) Some new aspects of creatine kinase $(\mathrm{CK})$ : compartmentation, structure, function and regulation for cellular and mitochondrial bioenergetics and physiology. Biofactors 8:229-234 